\title{
BIBECHANA
}

A Multidisciplinary Journal of Science, Technology and Mathematics ISSN 2091-0762 (Print), 2382-5340 (Online)

Journal homepage: http://nepjol.info/index.php/BIBECHANA

Publisher: Research Council of Science and Technology, Biratnagar, Nepal

\section{Correlation of solar wind velocity with different parameters during geomagnetic disturbances}

\author{
Sujan Dhakal ${ }^{1}$, Binod Adhikari ${ }^{1,2^{*}}$, Kiran Pudasainee ${ }^{2}$, Naryan Prasad Chapagain ${ }^{2,3}$ Drabindra \\ Pandit $^{1,2,3}$ Subodh Dahal ${ }^{4}$, Bikash Shrestha ${ }^{1}$, Bhawani Sapkota ${ }^{4}$, Daya Nidhi Chhatkuli ${ }^{5}$ \\ ${ }^{1}$ St. Xavier's College, Maitighar, Kathmandu, Nepal \\ ${ }^{2}$ Patan Multiple Campus, Patandhoka, Lalitpur, Nepal \\ ${ }^{3}$ Central Department of Physics, Tribhuvan University, Kirtipur, Kathmandu, Nepal \\ ${ }^{4}$ Department of Physics, Himalayan College of Geomatic Engineering and LRM, Kathmandu \\ ${ }^{5}$ Department of Physics, Tri-Chandra Multiple Campus, Kathmandu, Nepal \\ "E-mail: binod.adhi@gmail.com
}

Article history: Received 29 April, 2018; Accepted 04 November, 2018

DOI: http://dx.doi.org/10.3126/bibechana.v16i0.21575

This work is licensed under the Creative Commons CC BY-NC License.

https://creativecommons.org/licenses/by-nc/4.0/

\section{cc) (†) \&}

\begin{abstract}
We have studied the solar wind velocity and it's relation with solar wind pressure, southward component of IMF-Bz, solar wind temperature (Tsw), solar wind density (Nsw) and geomagnetic indices during different geomagnetic disturbances. During disturbed days, there is a fluctuation of energy and plasma inside the magnetosphere, which changes the parameters like pressure, velocity, IMF-Bz, SYM-H and AE indices. The solar wind velocity shows very remarkable relationship with pressure. There is weak connection of solar wind pressure with IMF-Bz, although it is more geoeffective.
\end{abstract}

Keywords: Geomagnetic storm; solar wind velocity; cross-correlation analysis.

\section{Introduction}

Geomagnetic disturbance is a temporary disturbance on earth magnetosphere initiated by various solar phenomena which produces abnormal condition in the interplanetary magnetic field (IMF) [1]. This disturbance may occur due to solar wind, coronal mass ejection (CME), solar flare, corotating interaction region (CIR) [2]. Solar wind is the continuous flow of plasma coming out from the sun [3]. It has the magnetic field which recombines with Earth's magnetic field. During the process of interaction energy and plasma are resettled into the magnetosphere and the magnetic field energy is converted into thermal energy and kinetic energy $[4,5]$. The Earth surrounding atmosphere is heated by the supersonic collision less magnetized plasma which carried a large amount of kinetic and electrical energy from solar wind. Some of this energy finds its way into our magnetosphere creating 
turmoil in geomagnetic activity resulting into geomagnetic storms, substorms as well as HILDCAAs (high intensity long duration continuous auroral activities) [6,7]. Generally, the most intense geomagnetic storms are due to CMEs whereas CIRs cause less intense geomagnetic storms [8]. The intensity of the geomagnetic storm depends upon the solar wind structures [9]. A typical geomagnetic storm is caused by a solar wind shock wave and/or cloud of magnetic field that interacts with the Earth's magnetic field [3]. Both interactions increase plasma movement through the magnetosphere that increase an electric current in the magnetosphere. The impact of different solar phenomenon is measured by solar wind parameters and geomagnetic indices. Geomagnetic storm cause changes in geomagnetic indices such as Disturbance storm time (Dst), Kp, AE etc. Geomagnetic indices are ground based measurements that are collected to quantify the state of the magnetosphere and it shows how the Earth reacts to given wind structure. These indices perturbation give the idea of the Earth's magnetosphere which is continuously affected by the solar wind flowing from the sun's corona [2, 10, 11]. Geomagnetic indices are the measure of the geomagnetic activity which plays the significance role in describing the space weather. Space weather refers to highly vulnerable conditions in the near earth geo-space environment. The geomagnetic indices are used to quantify the ability and geoeffectiveness of interplanetary solar wind structure to cause geomagnetic storms $[5,6]$. The increase in the solar wind pressure initially can compress the magnetosphere [11].

\section{Dataset and Methodology}

The data for this study have extracted the OMNI (Operating Mission as Nodes on the Internet) wave page and downloaded from the official website of NASA https://omniweb.gsfc.nasa.gov/data set for solar wind measurements.

\section{Cross-correlation}

Cross-correlation is simply a measure of statistical relationships involving two or more variables as a function of time lags applied to one of them. One can simply understand the cross correlation as a measure of similarities of two different time series function, one relative to the other. It is also known as sliding dot product or sliding inner product. Its value ranges from -1 to 1 and with the highest degree of cross correlation its value is close to 1 . It is the standard method to estimate the degree to which two different series are correlated.

\section{Result and Discussion}

In this section, we are dealing with cross correlation of solar wind velocity with component of IMF $(\mathrm{Bz})$ and geomagnetic indices for four different geomagnetic disturbance on different period. Mainly correlation between Vsw with solar wind parameter Psw, SYM-H and AE are analyzed. The brief discussion of the event using solar wind parameter and interplanetary parameters are discussed below.

\section{Event 1: January 20, 2015}

The case study presented in Figure 1 represents fluctuation in solar and interplanetary parameters during $20^{\text {th }}$ January 2015. The bottom panel of figure 1 shows the minimum and the maximum value of $\mathrm{AE}$ is 0 and $350 \mathrm{nT}$ respectively. Similarly, the minimum and maximum value of $\mathrm{SYM}-\mathrm{H}, \mathrm{Bz}$, Psw, Nsw, Vsw and Tsw are found to be $-19 \mathrm{nT}$ and $36 \mathrm{nT}, 7.82 \mathrm{nT}$ and $-5.95 \mathrm{nT}, 0.93 \mathrm{nPa}$ and 9.75 , $5 \mathrm{n} / \mathrm{cc}$ and $50.83 \mathrm{n} / \mathrm{cc}, 273.4 \mathrm{Km} / \mathrm{s}$ and $318 \mathrm{Km} / \mathrm{s}, 13849 \mathrm{~K}$ and $53979 \mathrm{~K}$. respectively. To be classified as storm, it must have SYM-H $\leq-50 \mathrm{nT}$ [3]. Thus, it is characterized as quiet day event studies the variation of Sq current over the various four quietest days of February 2003 and the results represents that $\mathrm{Sq}(\mathrm{H})$ exhibits transient variations with varying amplitude [12]. The analysis was carried out on solar quiet days using hourly values of horizontal $\mathrm{H}$ components of geomagnetic field. 
The variation of $\mathrm{Sq}-\mathrm{H}$ is characterized maximum around the daytime (07:00-17:00LT) and the minimum values during pre-sunrise hours between 05:00 and 06:00LT. It is clear that before the sunrise, conductivities are low due to absence of solar thermal heating. Due to lack of solar thermal heating causes the ionosphere conductivities to be the weakest pre-sunrise hours over all the day throughout the year. During a geomagnetic disturbance, there is an energy input inside the magnetosphere which changes atmospheric parameters. But during quiet periods, the disturbances measurements on the ground are less significant.
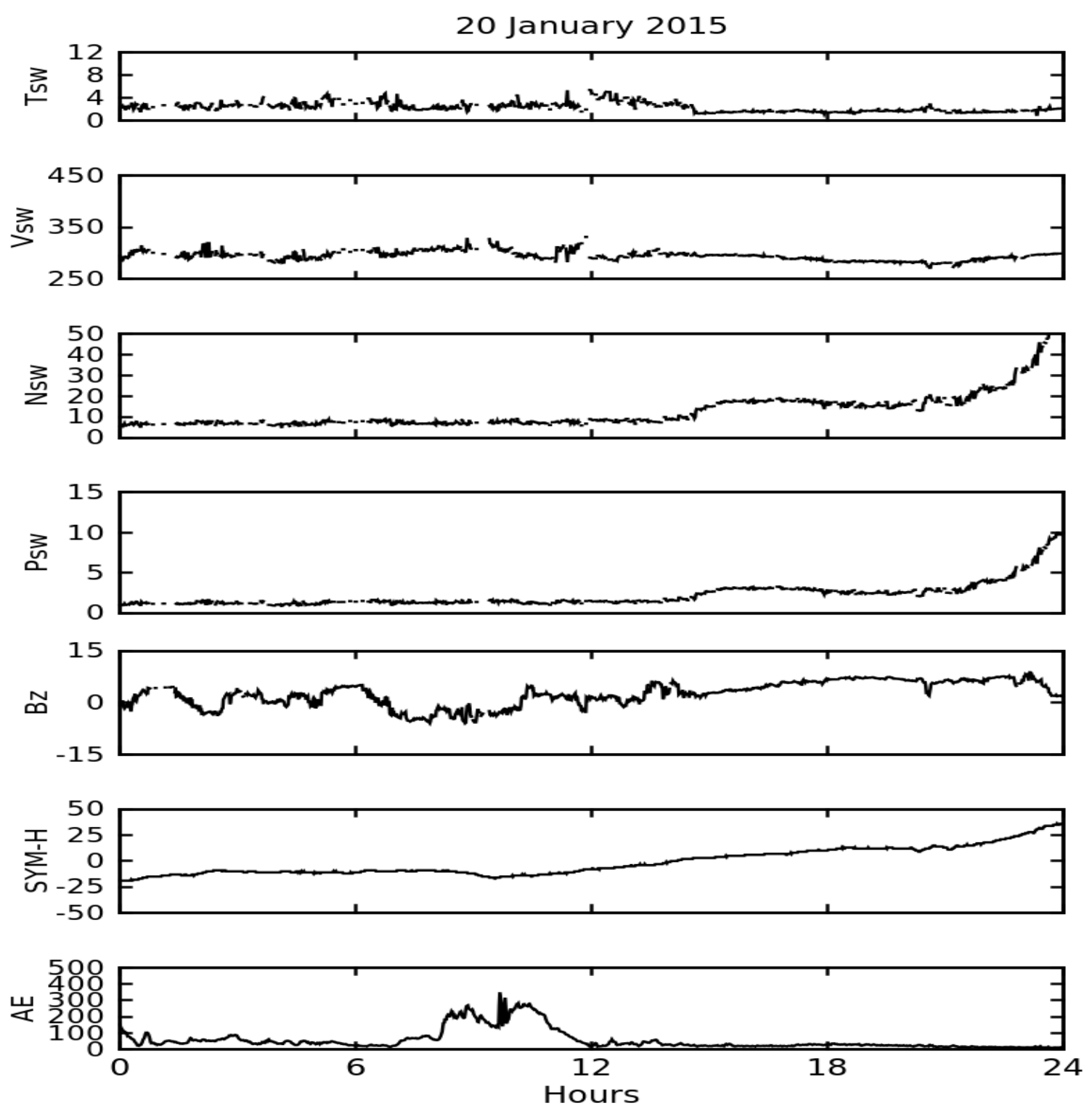

Fig. 1: From top to bottom, the panels shows the variations of the Tsw $\left(10^{4} \mathrm{~K}\right)$, Vsw $(\mathrm{Km} / \mathrm{s})$, Nsw (n/cc), Psw (nPa), Bz (nT), SYM-H (nT) and AE (nT) in GSM coordinate system respectively during $20^{\text {th }}$ January 2015 . 
Figure 2 shows the cross-correlation of Vsw with solar parameters and geomagnetic indices during 20th January 2015. The horizontal axis represents the scale in minutes and the vertical axis represents the cross-correlation coefficient. The red curve shows the correlation between Vsw and Tsw is highly positive with coefficient around 1. The sky-blue curve shows correlation between VswSYM-H is moderate and sometime crosses the zero points and reaches a negative value around -0.5 . The Psw-Bz (blue curve) shows positive correlation with correlation coefficient around 0.6 at time lag -800 minutes. Similarly, the Vsw-AE (pink curve) and Vsw-Nsw (yellow curve) shows positive correlation with correlation coefficient around +0.7 and +0.8 respectively. The Vsw-Psw (black curve) shows similar nature as yellow curve with correlation coefficient +0.8 . Here moderately and less correlated line seems to be more irregular and fluctuating than the highly correlated ones.

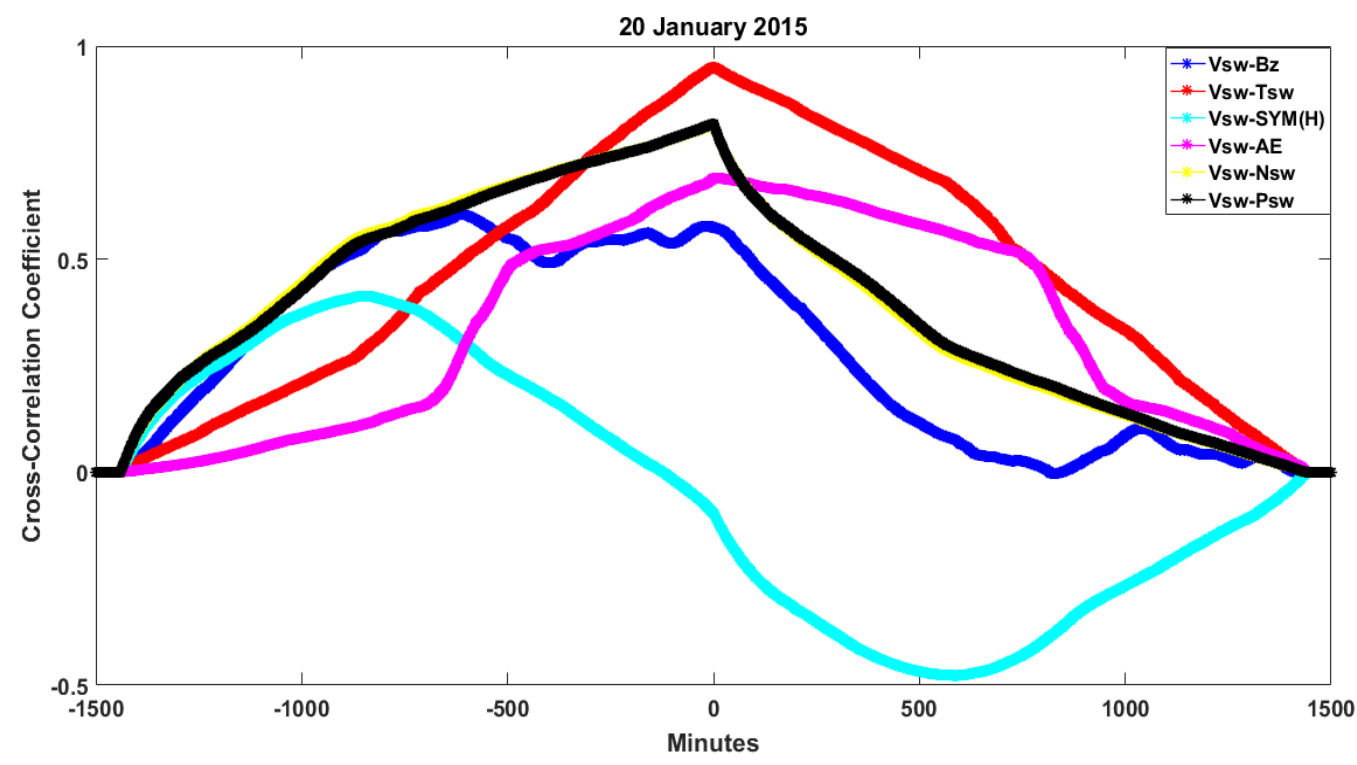

Fig. 2: Cross-correlation of solar wind velocity with southward component of interplanetary magnetic field (Bz), solar wind temperature (Tsw), SYM (H), AE solar wind density (Nsw), and solar wind pressure respectively during $20^{\text {th }}$ January 2015.

\section{Event 2: 6-10 June 2006}

Figure 3 represents the fluctuation of various parameters during 6 to 10 June 2006. Here, the AE has the minimum value around $23 \mathrm{nT}$ at 3:00 UT and the maximum value around $1191 \mathrm{nT}$ at 6:00 UT. Similarly, the minimum and maximum value of SYM-H is $-45 \mathrm{nT}$ and $16 \mathrm{nT}$ respectively. The maximum value of $\mathrm{Bz}$ component (GSM) is $10.62 \mathrm{nT}$ and minimum $\mathrm{Bz}$ component is $-12.02 \mathrm{nT}$. The flow pressure lies between the range $1.01 \mathrm{nPa}$ to $18.26 \mathrm{nPa}$. The maximum value of proton density $51.78 \mathrm{n} / \mathrm{cc}$ and the minimum value is $1.39 \mathrm{n} / \mathrm{cc}$. The solar wind speed fluctuates between $327.9 \mathrm{~km} / \mathrm{s}$ and $677.7 \mathrm{~km} / \mathrm{s}$ and the solar wind temperature fluctuates between $27705 \mathrm{~K}$ and $192066 \mathrm{~K}$. The figure 3 indicates that the HILDCAA events starts at the mid-day of $6^{\text {th }}$ June 2006 and continue till the beginning of $9^{\text {th }}$ June 2006. During the event there is fluctuation in the values of parameters Vsw, Bz, Psw, Nsw, SYM-H and AE respectively. The result obtained for this event are satisfied the criteria for HILDCAA as suggested by Tsurutani and Gonzalez (1987). In 1995, Tsurutani et al. has found that 
the recovery phase is longer in the year 1974.The Dst recoveries are associated with high AE values. The main reason for this is high fluctuation in interplanetary $\mathrm{Bz}$ in the high velocity stream. With every instant of southward field turnings, there is an increase in AE and decrease in Dst. The southward turnings cause magnetic reconnection and plasma injections into the night side magnetosphere. There is slight decrease in Dst at each of these injections. These periods of continuous substorm activity are defined as HILDCAA. The main cause of the interplanetary Bz fluctuations is given by Belcher and Davis (1971). They have demonstrated that Alfven waves are propagating away from the sun which are present in the high-speed streams leads to the Bz fluctuations within the CIRs leading to the irregular shaped storm main phase, and also the fluctuations that cause HILDCAAs in the storm "recovery phases". [7] Has described the seasonal dependence of HILDCAA events to determine the solar cycle. Their study clarifies that the occurrence of HILDCAA events during the different phases of the solar cycle. That paper also discussed the interplanetary Alfven waves by indicating high IMF BZ variance and normalized variance of the HILDCAA events. [12,13] studied the solar wind energy during HILDCAA in his $\mathrm{PhD}$ thesis to described the electrodynamical process caused by the solar wind magnetosphere coupling for HILDCAA events (high intensity, long duration continuous AE activity) $[12,14]$. He has also described the effect of HILDCAA event on geomagnetic status and its consequence on Earth's environment by analysing the coupling mechanism between the solar wind and magnetosphere.

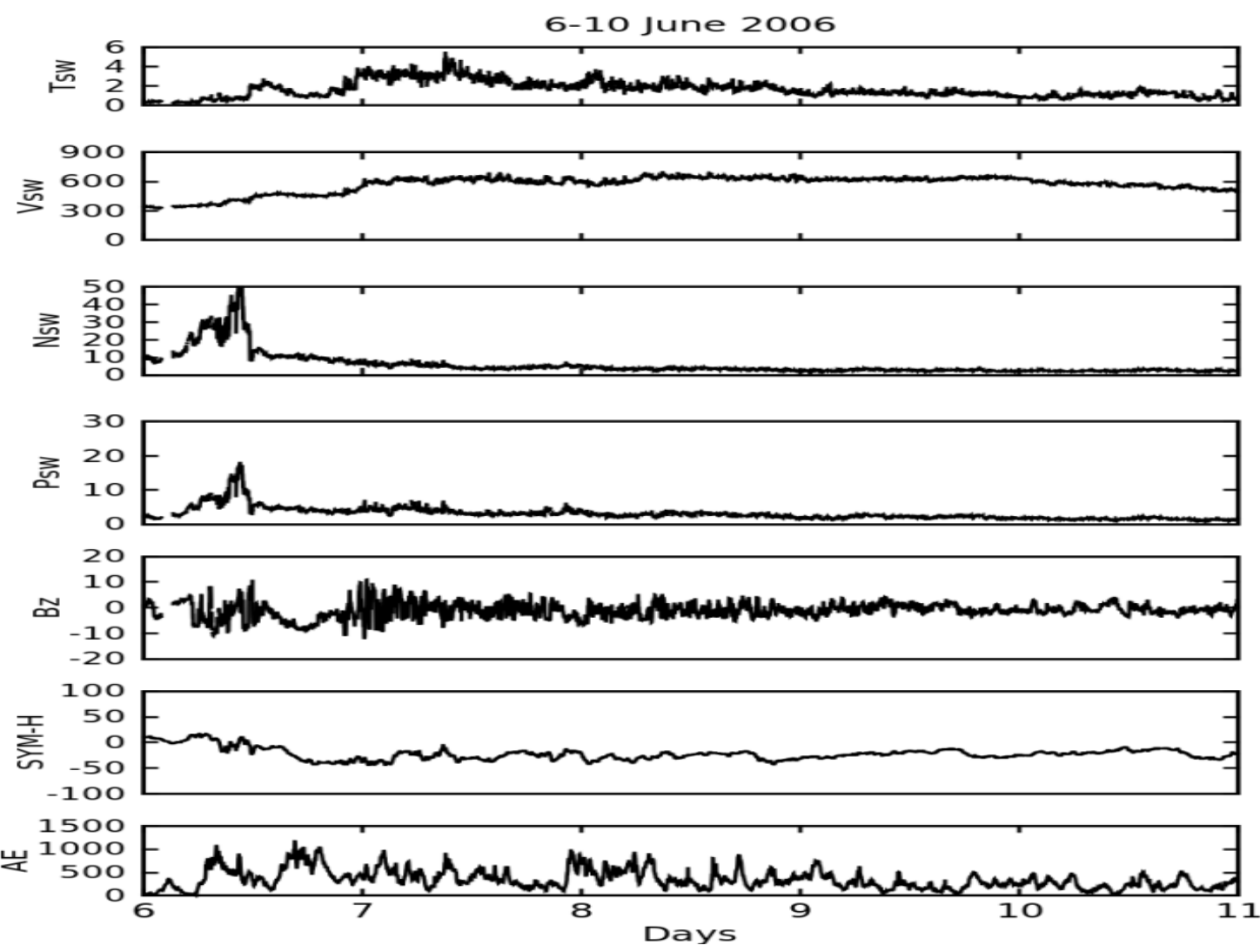

Fig. 3: From top to bottom, the panels shows the variations of the Tsw $\left(10^{4} \mathrm{~K}\right), \mathrm{Vsw}(\mathrm{Km} / \mathrm{s})$, Nsw $(\mathrm{n} / \mathrm{cc}), \mathrm{Psw}(\mathrm{nPa}), \mathrm{Bz}(\mathrm{nT}), \mathrm{SYM}-\mathrm{H}(\mathrm{nT})$ and AE (nT) in GSM coordinate system respectively during 6-10 June 2006. 
Figure 4 shows the cross-correlation of Vsw with solar parameters and geomagnetic indices during 610 June 2006. The horizontal axis represents the scale in minutes and the vertical axis represents the cross-correlation coefficient. In this figure, the sky-blue curve shows negative cross-correlation coefficient of around -0.90 between SYM-H-Vsw with a time lag around 10 minutes and it does not show any positive correlation during this event. The negative correlation signifies that if the value of Vsw decreases with time then SYM-H increases with increasing time and vice-versa. This means they have a negative relationship with each other. Similarly, red curve shows positive correlation between Vsw-Psw with high correlation coefficient of about 0.9. The Vsw-AE pink curve has a good positive cross-correlation with correlation coefficient of about 0.78 at zero-time lag. Similarly, the Vsw-Psw black curve and Vsw -Nsw yellow curve also shows good positive cross-correlation with correlation coefficient of about 0.76 and 0.60 at 0 -time lag respectively. Blue curve ( Vsw-Bz) shows negative correlation with correlation coefficient of about -0.3 at 0 minutes time lag.

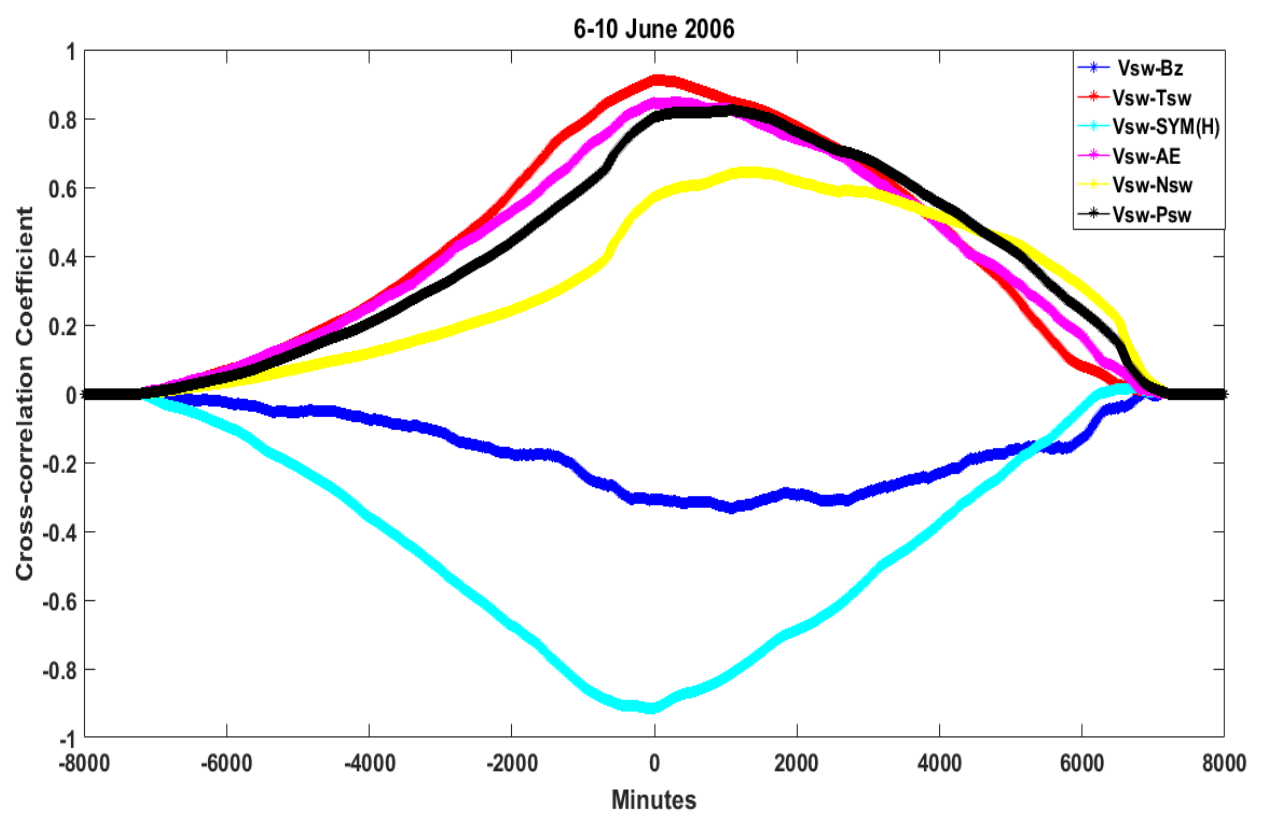

Fig. 4: Cross-correlation of solar wind velocity with southward component of interplanetary magnetic field $(\mathrm{Bz})$, solar wind temperature (Tsw), SYM(H), AE solar wind density (Nsw), and solar wind pressure respectively during (6-10) June 2006.

\section{Event 3: 23 June 2015}

Figure 5 depicts the same parameters fluctuation as above during 23 June 2015. Here, it is clearly seen the three phases of a geomantic storm. The initial phase begins early hours. The main phase of the storm begins from 2:00 to 12:00 UT. Finally, the recovery phase begins from 13:00 and end at 24:00 UT. During main phase, the value of plasma speed (Vsw) is around $700 \mathrm{~km} / \mathrm{s}$ and the value of Nsw lies between $0 \mathrm{n} / \mathrm{cc}$ to $30 \mathrm{n} / \mathrm{cc}$ and it is maximum around 1:00 UT. The value of Bz shows variation between $-20 \mathrm{nT}$ to $20 \mathrm{nT}$. The value of Psw is around $10 \mathrm{nPa}$ and the value of SYM-H varies between $200 \mathrm{n}$ T to $-50 \mathrm{n}$ T. The minimum value of SYM-H $-200 \mathrm{nT}$ suggested by Gonzalez et al. 1992 indicates this event is an intense geomagnetic storm. Here we have observed in SYM-H with large negative value of -200nT with peak value. During this instant the solar wind speed and IMF Bz has maximum value of $800 \mathrm{Km} / \mathrm{s}$ and $-20 \mathrm{nT}$ respectively. The value of $\mathrm{AE}$ is around $1600 \mathrm{nT}$. This event is 
due to the direct dependence of the geomagnetic storm with south-north component of magnetic field Bz. According to Gonzalez et al. (1992), the intense interplanetary field can be thought of a highspeed stream, the intrinsic ejecta (called driver gas fields) and the shocked and compressed fields and plasma due to the collision of the high-speed stream with the slower solar wind preceding it. The greater the relative velocity, the stronger is the shock and the field compression, had investigated the effect of solar wind dynamic pressure Psw, and preconditioning over 80 large magnetic storms (Dst<100nT) which was occurring during solar cycle 23, and found that in the main phase of a storm, when there is a large enhancement of the dynamic pressure, there is always an increase in the Dst peak value during the storm main phase, the weak Psw verses Vsw relationship was connected to the ring current energy losses which may be originated from different sources. The solar wind dynamic pressure has a strong relationship with the southward IMF Bz during the main phase of a storm, typically when the plasma flow speed value is very large, exceeding the $650 \mathrm{~km} / \mathrm{s}$ value, and the Dst<100nT.
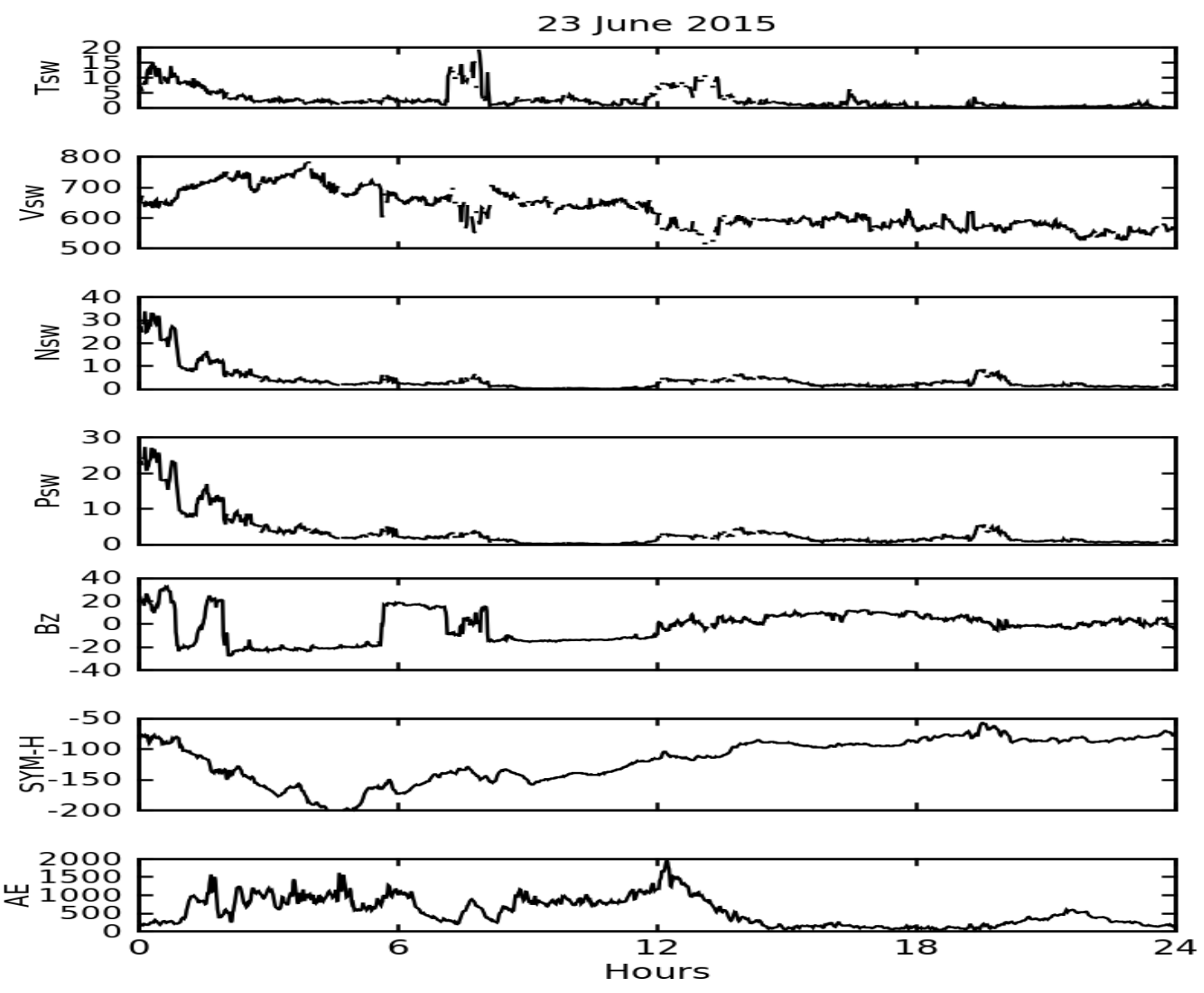

Figure 5: From top to bottom, the panels shows the variations of the Tsw $\left(10^{4} \mathrm{~K}\right)$, Vsw $(\mathrm{Km} / \mathrm{s}), \mathrm{Nsw}$ $(\mathrm{n} / \mathrm{cc}), \mathrm{Psw}(\mathrm{nPa}), \mathrm{Bz}(\mathrm{nT}), \mathrm{SYM}-\mathrm{H}(\mathrm{nT})$ and AE (nT) in GSM coordinate system respectively during 23 June 2015. 
Figure 6 represents the cross-correlation of Vsw with solar parameters and geomagnetic indices during 23 June 2015. Here the horizontal axis represents the scale in minutes and the vertical axis represents the cross-correlation coefficient. The pink curve shows the correlation between AE and Vsw. This curve represents strong positive cross-correlation coefficient of about 0.8 at lag zero. The sky-blue curve shows good correlation between Vsw-SYM-H with cross-correlation coefficient of around -1 at lag of zero minutes. The negative correlation signifies that if the value of Vsw decreases with time then SYM-H increases with time and vice-versa. The blue curve of Vsw-Bz represents uncorrelated. The red curve represent correlation between Vsw-Tsw and it shows good correlation with correlation coefficient of about 0.75 at lag zero. Similarly, the correlation between Vsw-Nsw and Vsw-psw are represented by yellow and black color respectively. They shows good positive crosscorrelation of correlation coefficient about 0.6 at zero lag.

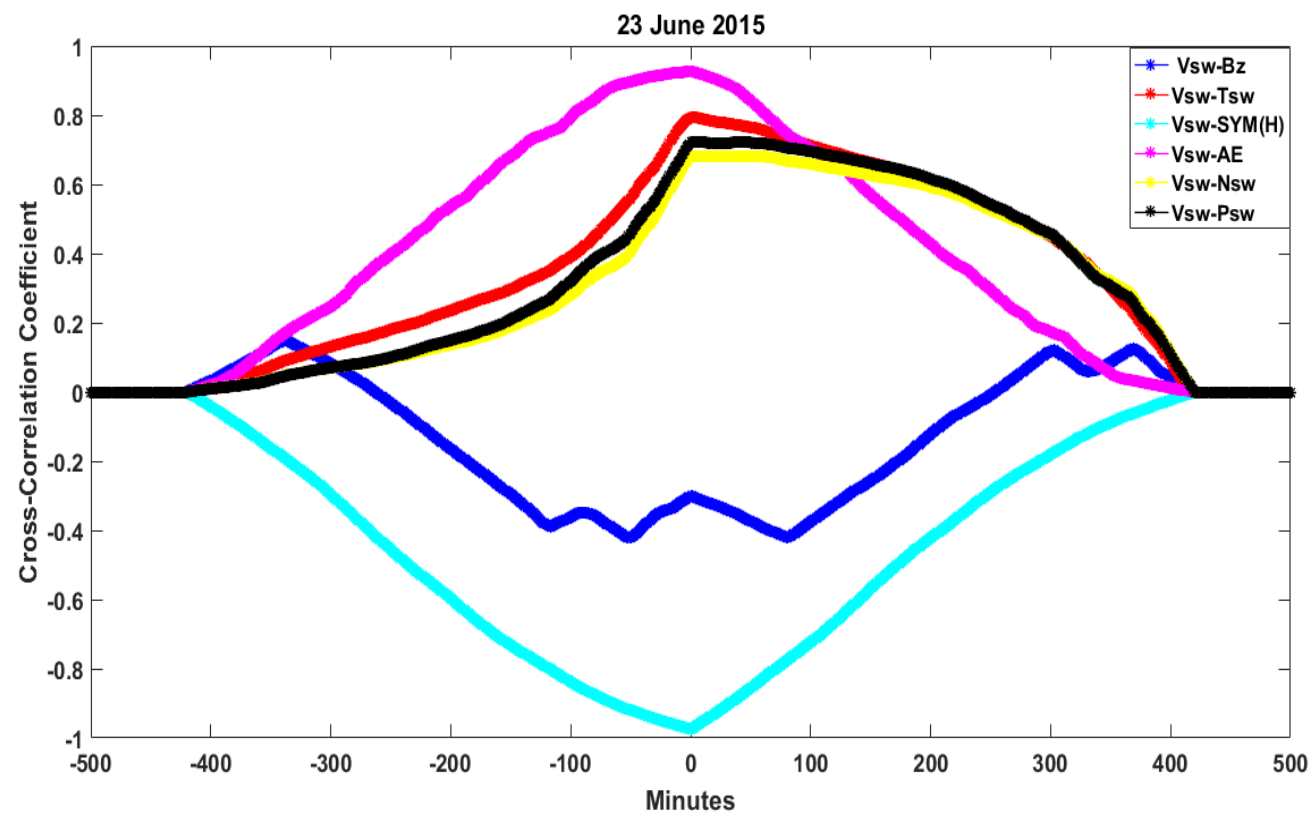

Fig. 6: Cross-correlation of solar wind velocity with southward component of interplanetary magnetic field $(\mathrm{Bz})$, solar wind temperature (Tsw), SYM(H), AE solar wind density $(\mathrm{Nsw})$, and solar wind pressure respectively during 23 June 2015.

\section{Event 4: 01 March 2011}

Figure 7 depicts the same parameters fluctuation as above during $1^{\text {st }}$ March 2011. Here, it shows that the minimum SYM-H index for this event is $-71 \mathrm{nT}$ and the maximum SYM-H index is $23 \mathrm{nT}$. The $\mathrm{AE}$ index range in between the minimum value of $18 \mathrm{nT}$ and the maximum value is $1348 \mathrm{nT}$. The maximum $\mathrm{Bz}$ component (GSM) is $16.16 \mathrm{nT}$ and minimum $\mathrm{Bz}$ component is $-15.01 \mathrm{nT}$. The flow pressure lies between the range $3.63 \mathrm{nPa}$ to $9.47 \mathrm{nPa}$. The maximum value of proton density 44.91 $\mathrm{n} / \mathrm{cc}$ and the minimum value is $4.07 \mathrm{n} / \mathrm{cc}$. Similarly, the speed of solar wind is in between $301.0 \mathrm{Km} / \mathrm{s}$ to $680.4 \mathrm{Km} / \mathrm{s}$. and temperature of solar wind varies in the range $21910 . \mathrm{K}$ to 5771.35 . K respectively. The solar wind parameters and interplanetary magnetic field fluctuation represent this is a moderate sub storm. The growth phase begins around 8:00 UT of $1^{\text {st }}$ March 2011. The expansion phase of the substorm begins from 12:00 UT of the same day. Finally, the recovery phase begins from 20:00 UT. 
The solar wind parameters, interplanetary magnetic field fluctuation show low values, characterizing this as moderate sub-storm. During the period of substorm the Dst and IMF Bz has peak value of -

50nT and $15.01 \mathrm{nT}$ respectively whereas other parameters such as solar wind speed, and AE has maximum value. The increases in the Dst and the SYM H indices after substorm onsets are related to the depolarization. This shows that about one third of the northward equatorial magnetic field variations are associated with variations in the solar wind pressure, a second third with substorm onsets, and the final third with interplanetary magnetic field reorientations in the north-south direction. Substorms are fundamental and dynamic processes in the magnetosphere, converting captured solar wind magnetic energy into plasma energy $[13,14,15]$. These substorms have been suggested to be a key driver of energetic electron enhancements in the outer radiation belts. The storage and release of energetic electron during substorms in the radiation belt leads to characteristic changes in auroral morphology and emission intensity in the polar ionosphere and high-latitude surface magnetic field $[16,17,18]$. The occurrence rate of substorms is more frequent and more intense during geomagnetic storms [3]. The typical duration of substorm is about 2-4 hours. The length of growth, expansion and recovery phases vary from the short expansion of 15-20 min to a lengthy growth or recovery of a few hours $[19,20,21]$.

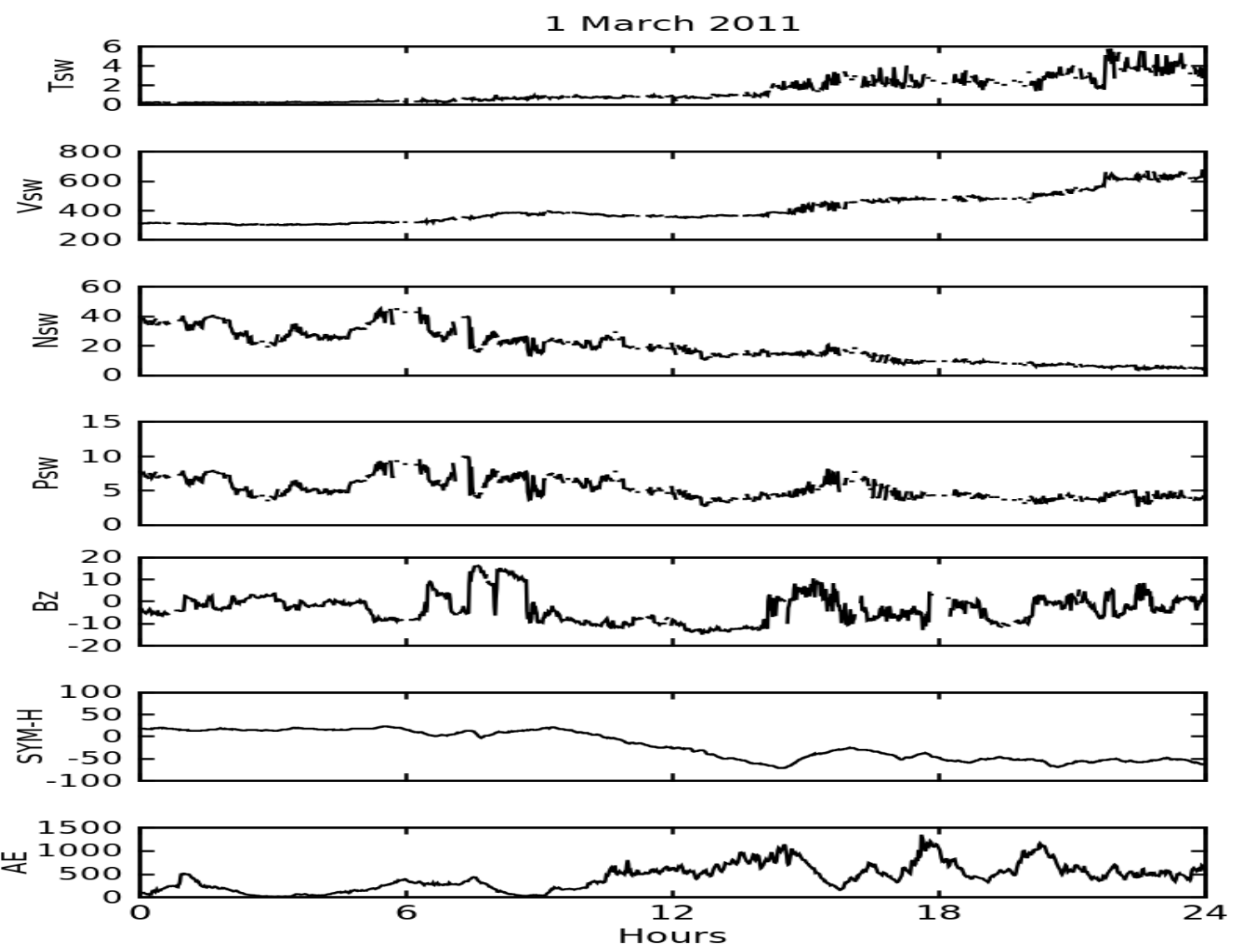

Fig. 7: From top to bottom, the panels shows the variations of the Tsw $\left(10^{4} \mathrm{~K}\right)$, Vsw $(\mathrm{Km} / \mathrm{s})$, Nsw (n/cc), Psw (nPa), Bz (nT), SYM-H (nT) and AE (nT) in GSM coordinate system during respectively during $1^{\text {st }}$ March 2011. 
Figure 8 the cross-correlation of Vsw with solar parameters and geomagnetic indices during 23 June 2015. Here the horizontal axis represents the scale in minutes and the vertical axis represents the cross-correlation coefficient. The black curve shows the correlation between Vsw and Psw. The curve shows strong positive cross-correlation coefficient of about 0.9 at lag zero. The sky-blue curve shows correlation between Vsw-SYM-H is moderate and sometime crosses the zero points. The correlation between Vsw-SYM-H is represented by pink curve shows high negative cross-correlation coefficient of around 0.8 at lag of -20 minutes. The negative correlation signifies that if the value of Vsw decreases with time then SYM-H increases with increasing time and vice-versa. The blue curve of BzVsw represents there week correlation. The correlation between Vsw-Nsw (yellow curve) shows good correlation with correlation coefficient about 0.85 at lag zero. Similarly, pink curve also shows good positive cross-correlation between Vsw-AE with correlation coefficient of about 0.8 at zero lag. The correlation between Vsw and Tsw is positive with correlation coefficient of about 0.80 at lag zero.

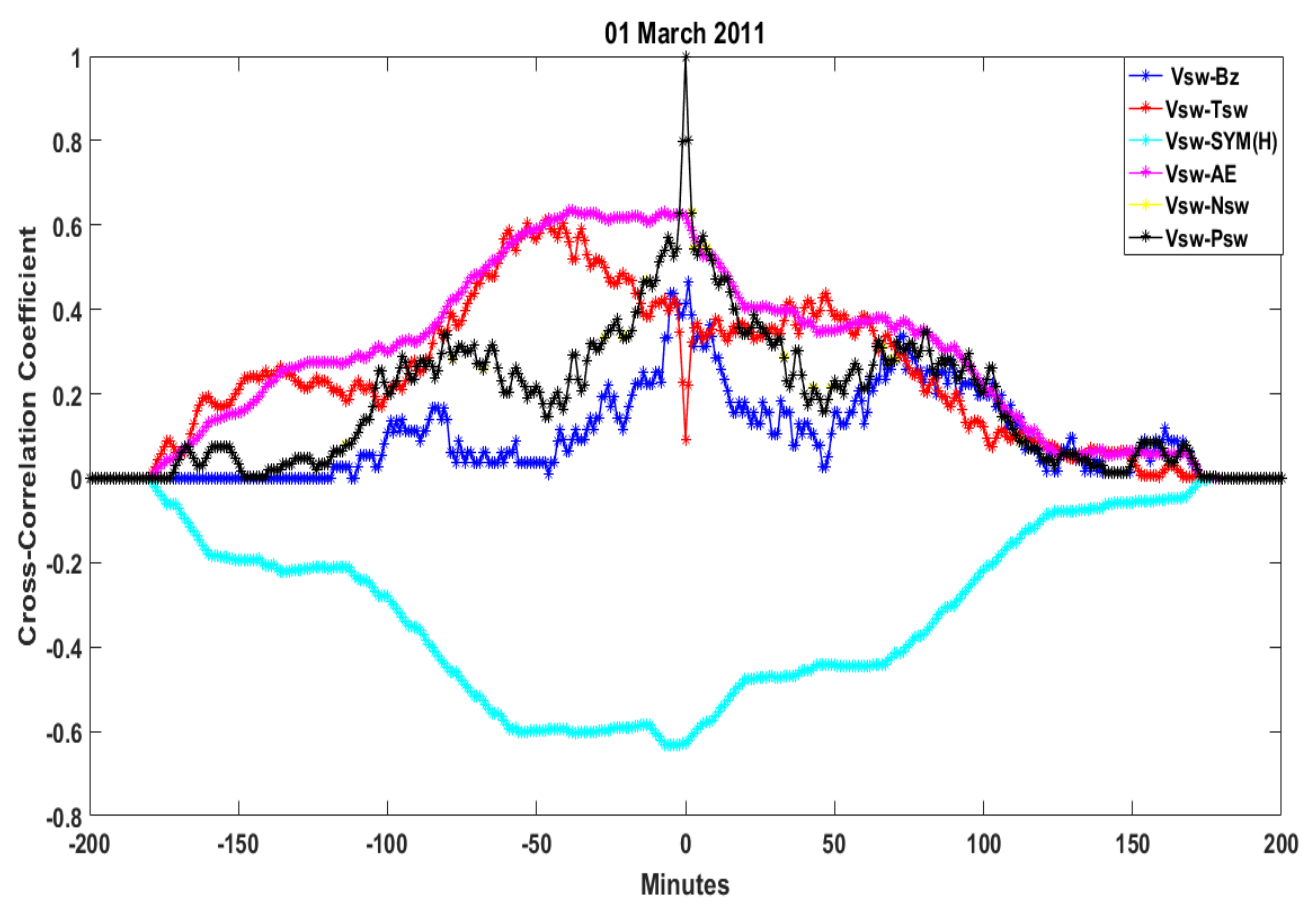

Fig. 8: Cross-correlation of solar wind velocity with southward component of interplanetary magnetic field $(\mathrm{Bz})$, solar wind temperature $(\mathrm{Tsw}), \mathrm{SYM}(\mathrm{H}), \mathrm{AE}$ solar wind density $(\mathrm{Nsw})$, and solar wind pressure respectively $1^{\text {st }}$ march 2011.

\section{Conclusion}

In this study, we have studied correlation of solar wind velocity with different solar and interplanetary indices during four different geomagnetic disturbances. This study shows the major sources of the geomagnetic disturbance are recognized to be CME, CIR, etc. The interaction of solar wind component with Earth's magnetosphere can cause geomagnetic storms, substorms, supersubstorms, HILDCAA, etc. The conclusions are summarized as the follows:

- During disturbed days, there is a fluctuation of energy and plasma inside the magnetosphere, which changes the parameters like pressure, plasma density, velocity, IMF-Bz SYM-H and AE indices. 
- The solar wind velocity shows positive correlation with pressure and negative correlation with $\mathrm{SYM} / \mathrm{H}$ in every event.

- There is weak connection of solar wind pressure with IMF-Bz, although it is more geoeffective.

- The strong correlation between solar wind pressure and velocity during storms initial phase was linked to the accelerated ring current build-up.

\section{Acknowledgements}

The data sets for this study were downloaded from NASA website (http://omniweb.gsfc.nasa.gov/ow_min.html. and www.cosmic.ucar.edu/). We would like to thank NASA.

\section{References}

[1] B.T. Tsurutani, W.D. Gonzalez, F. Tang, S. Akasofu, and E. Smith, Origin of interplanetary southward magnetic fields response for major magnetic storms near solar maximum (1978-1979), J. Geophys. Res. A8 (1988) 8519 - 8531. doi.org/10.1029/JA093iA08p08519.

[2] J. T. Gosling, Coronal mass ejections and magnetic flux ropes in interplanetary space, Geophys. Monog. 58 (1990) 343-364. doi.org/10.1029/GM058p0343.

[3] W. D. Gonzalez, J. A. Joselyn, Y. Kamide, H. W. Kroehl, G. Rostoker, B. T. Tsurutani, V. M. Vasyliunas, What is a Geomagnetic Storm? J. Geophys. Res, 99 ((1994) 5771-5792. doi.org/10.1029/93JA02867.

[4] S.I. Akasofu, Solar-wind disturbances and the solar wind-magnetosphere energy coupling function, Space Sci. Rev. 34 (1983) 173-183. doi.org/10.1007/BF00194625.

[5] Elliott, H. A., J.-M. Jahn, and D. J. McComas (2013), The Kp index and solar wind speed relationship: Insights for improving space weather forecasts, Space Weather, 11(2013)339-349 doi.org/10.1002/swe.20053.

[6] B. Adhakari, HILDCAA-Related Effects Recorded in Middle-Low Latitude Magnetometers. PhD Doctoral Dissertation São José dos Campos, 12, 14, 17 18, 21, 22, 23, 31, 34, 35, 36, 38, 45, (2015).

[7] R. Hajra, E. Echer, B. T. Tsurutani, and W. D. Gonzalez, Solar cycle dependence of High-Intensity LongDuration Continuous AE Activity (HILDCAA) events, relativistic electron predictors?, J. Geophys. Res. Space Physics 118 (2013) 5626-5638. doi.org/10.1002/jgra.50530.

[8] K. Mursula and B. Zieger, The 13.5-day periodicity in the sun, solar wind and geomagnetic activity: The last three solar cycles, J. Geophys. Res. 101, 27, 077-27, 090, 1996. doi.org/10.1002/jgra.50530.

[9] B. T. Tsurutani, R. L. McPherron, W. D. Gonzalez, Lu. Gang, N. Gopalswamy, and F. L. Guarnieri, Magnetic Storms Caused by Corotating Solar Wind Streams, AGU Geophys. Monogr. Ser., 167(2006). doi.org/10.1029/167GM03.

[10] T. N. Davis and M. Sugiura, Auroral electrojet activity indexes AE and its universal time variations, J. Geophys. Res. 71(3) (1966) 785-801. doi.org/10.1029/JZ071i003p00785.

[11] S. Chapman, Notes on the solar corona and the terrestrial ionosphere, smithsonian contr., Astrophys., 2, (1957) 1.

[12] B. Adhikari, P. Baruwal, and N. P. Chapagain (2016), Analysis of supersubstorm events with reference to polar cap potential and polar cap index, Earth and Space Science, 3. doi.org/10.1002/ 2016EA000217.

[13] S.I Akasofu, The Growth of the Storm-Time Radiation Belt and the Magnetospheric Substorm, J. Geophys. Res. 15(1-2) (1968) 7-21. doi.org/10.1111/j.1365-246X.1968.tb05741.x.

[14] S. I. Akasofu and S. Yoshida, 'Growth and decay of the ring current and the polar electrojets' J. Geophys. Res. 71(17) (1966) 4211-4212. doi.org/10.1029/JZ071i001p00231. 
[15] C. Forsyth, I. J. Rae, K.R. Murphy, What effect do substorms have on the content of the radiation belts? Journal of Geophysical Research Space Physics 121(7) (2016) 6292-6306. doi.org/10.1002/2016JA022620.

[16] E.I. Tanskanen, Terrestrial substorms as a part of global energy flow, Ph.D. dissertation, Univ. of Helsinki, Helsinki, Finland. 2002.

[17] J. W. Gjerloev, R. A. Hoffman, The large-scale current system during auroral substorms. J. Geophys. Res. 119(6) (2014) 4591-4606. doi.org/10.1002/2013JA019176.

[18] W. D. Gonzalez, B. T. Tsurutani, L. Alicia and G.D. Clia, Interplanetary origin of geomagnetic Storms, Space Science Reviews 88 (1999) 529. doi.org/10.1023/A:1005160129098.

[19] E .N. Parker, Dynamics of the interplanetary gas and magnetic fields, Astrophys. J. 128 (1958) 664-675. doi.org/10.1086/146579.

[20] C.W. Snyder, M. Neugebauer, and U.R. Rao, The solar wind velocity and its correlation with cosmic ray variations and with solar and geomagnetic activity. J. Geophys. Res. 68 (1963) 6361. doi.org/10.1029/JZ068i024p06361.

[21] W. Chin-Chun, R. P. Lepping, Effects of magnetic clouds on the occurrence of geomagnetic storms: The first 4 years of Wind J. Geophys. Res., 107, Issue A10 (2002). doi.org/10.1029/2001JA000161. 